\title{
Egyptian Checklist of Mites from Aromatic, Medicinal and Ornamental Plants
}

\author{
Amal H.M. Romeih ${ }^{*}$; M.F. Hassan ${ }^{\star}$; Marguerite A. Rizk ${ }^{*}$ \\ and Reham I.A. Abo-Shnaf* \\ *Agric. Zoology and Nematology Dept. Faculty of Agric., Cairo Univ., Giza, Egypt \\ "* Vegetable and Ornamental Acarology Dept., Plant Protection Research Institute, Agricultural Research Centre, Giza, \\ Egypt, rehamaboshnaf@yahoo.com \\ (Received: July17, 2010)
}

\begin{abstract}
A survey of mites associated with 25 aromatic and medicinal plant species of 13 families i.e., Araliaceae, Caricaceae, Chenopodiaceae, Compositae, Ephorbiaceae, Lamiaceae, Lauraceae, Liliaceea, Meliaceae, Myrtaceae, Papaveraceae, Rutaceae and Umbelliferae; addition to 75 ornamental plant species belonging to mine groups (cut-flower rose, flowering bulbs, flowering potted plants, ornamental trees, shrubs, vines, fences, ornamental palm trees and cut flowers) were carried out for one year. Thirty-eight families including 92 genera and 168 mite species were collected from these plant species at Giza and Fayoum Governorates. One new species of the family Phytoseirdae from genus Euseius was recorded. Twenty-four species belonging to different families; Acaridae (seven species), Parasitidae (six species), five species to each family Cunaxidae and Cheyletidae, and one species to the family Tarsonemidae were furstly recorded.
\end{abstract}

Key Words: Survey, Mite pests, Predacious mites, Ornamental plants, Aromatic plants, Medicinal plants, Giza, Fayoum.

\section{INTRODUCTION}

Production of ornamental flowers combines economic returns with satisfaction to the grower, and has recently experienced expressive growth. Some mite species feed on leaves, stems, flowers and even on the fruits. Others are found only underground where they feed on roots, tubers, or rhizomes. If the infestation is severe, leaves may fall and the number of flowers produced may be reduced considerably (Gallo et al., 2002). In addition to yield reduction, there may be a reduction in quality of the flowers (Karlik et al., 1995).

Some mites are predators feed on harmful insects and mites while others are fungivorous, saprophagous or of debatable feeding habit.

Aromatic and medicinal plants, herbs and spices are important source, for the synthesis of more complicated flavors, fragrances, pharmaceuticals, vitamins and other important chemicals. The survey of these plants and mite species associated with has been extensively studied in different countries as presented by Hanna et al., 1981; Mohamed \& Hassan, 1984; Palmer \& Pullen, 1995; Friend et al., 1999; Fan et al., 2000; Moraes et al., 2004; Cui et al., 2005; Jesse et al., 2006; Sallam et al., 2007; Amro, 2007; Rosenheim \& Fournier, 2007 and Lawson-Balagbo et al., 2008. Hence, the present study was pursued to survey acarine species inhabiting some ornamental, aromatic and medicinal plants at Fayoum and Giza Governorates in Egypt.

\section{MATERIALS AND METHODS}

Acari surveys of different ornamental, aromatic as well as medicinal plants are performed at Giza and Fayoum Governorates. Random samples of leaves, debris, litters and soil were collected periodically at fortnight intervals from February 2006 through January 2007. Ten leaves were taken from different levels of the plants throughout the growing season. However soil, debris and litter samples under plants were taken, then mites were extracted by modified Tullgren funnel. Phytophagous, predacious and other mites of miscellaneous feeding habits were identified.

\section{RESULTS AND DISCUSSION}

The number of plants sampled in each region varied according to their availability. A total of 25 aromatic and medicinal plant species in 13 families were sampled i.e., Hedera helix (Araliaceae); Carica papaya (Caricaceae); Chenopodium ambrosioides (Chenopodiaceae); Ambrosia maritima, Calendula officinalis and Matricaria chamomilla (Compositae); Ricinus communis (Ephorbiaceae); Mentha piperita, M. viridis, Ocimum basilicum, Origanum marjorana, Rosmarinus officinale and Salvia officinalis (Lamiaceae); Laurus nobilis (Lauraceae); Ruscus aculeatus (Liliaceae); Azadirachta indica (Meliaceae); Eucalyptus globules (Myrtaceae); Papaver somniferum (Papaveraceae); Citr aurantium (Rutaceae) and Ammi majus, Anethum graveolins, Apium graveolens, Coriandrum sativum, 
Foeniculum vulgare and Petroselinum sativum (Umbelliferae).

Also 75 omamental plant species of nine groups were sampled for mite species:

\section{Cut-flower rose: Rosa hybrida (Rosaceae).}

2. Flowering bulbs: Crinum asiaticum (Amaryllidaceae), Polianthes tuberose (Liliaceae) and Strelitzia reginae (Streliziaceae).

3. Flowering potted plants: Begonia sp. (Begoniaceae) and Pelargonium zonale (Geraniaceae).

4. Ornamental trees: Dracaena fragrans (Agavaceae); Monstera deliciosa (Araceae); Schefflera actinophylla (Araliaceae); Araucaria excelsa (Araucariaceae); Amphilobium paniculata and Spathodea nilotica (Bignoniaceae); Cassia nodosa (Caesalpinaceae); Casuarina equsetifolia (Casuarinaceae); Cupressus sempervirens (Cupressaceae); Acacia saligna, Bauhinia hookeru, Delonix regia, Dickrostachys nutans and Erythrina variegate (Leguminosae); Ficus benghalensis, F. nitida, F. sycamores, Morus alba and M. nigra (Moraceae), Callistemon citrinus (Myrtaceae); Pinus halepensis (Pinaceae); Phyllostachs pubescens (Poaceae); Salix babylanica (Salicaceae); Koelreuteria panniculata (Sapindaceae); Poulownia tomentosa (Scrophulariaceae) and Taxiodium distichum (Taxodiaceae).

5. Shrubs: Yucca gloriosa (Agavaceae); Nerium oleander and Plumeria alba (Apocynaceae); Acalyp $\hbar$ a marginta, A. wilkesiana and Euphorbia pulcherrima (Euphorbiaceae); Legerstroemia indica (Lythraceae); Hibiscus sabderiffa (Malvaceae) and Jasminum sambac (Oleaceae).

6. Vines: Syngonium podophyllum (Araceae), Tecoma stans (Bignoniaceae), Quisqualis indica (Combretaceae), Ipomoea palmate (Convolvulaceae), Bougainvillea spectabilis (Nyctaginaceae), Jasminum grandiflorum (Oleaceae) and Clerodendrum splendens (Verbenaceae).

7. Fences: Adhatoda vasica (Acanthaceae), Codiaeum variegatum (Euphorbiaceae), Murraya exotica (Mutaceae), Melaleuca genistifolia (Myrtaceae), Pittosporum tobira (Pittosporaceae), Dodonaea thunbergiana (Sapindaceae) and Lantana camara
(Verbenaceae).

8. Ornamental palm trees: Cycas revolute (Cycadaceae); Caryota mitis, Phoenix canariensis, $P$. dactylifera, Roystonia ragia and Washingtonia filifera (Palmaceae).

9. Cut flowers: Celosia pyramidalis (Amarantaceae); Vinca rosea (Apocynaceae); Gerbera jamesonii and Helianthus annus (Compositae); Alyssum maritimum and Matthiola incana (Cruciferae); Salvia leucantha (Lamiaceae); Agapanthus umbellatus and Cordyline fruticosa (Liliaceae); Althaea rosae (Malvaceae); Antirrhinum majus (Scrophulariaceae); Petunia hybrid (Solanaceae); $T$. majus majus (Tropaeolaceae) and Viola tricolor (Violaceae).

The list presented below comprises a total of 168 mite species from four orders; representing 92 genera of 38 families were collected from different plants. Nineteen of these species belong to two phytophagous families Tenuipalpidae and Tetranychidae and 93 predatory species belonging to the families Ascidae, Bdellidae, Caligonellidae, Camerobiidae, Cheyletidae, Cunaxidae, Digamasellidae, Eupalopsellidae, Eupodidae, Laelapidae, Macrochelidae, Ologamasidae, Pachylaelapidae, Parasitidae, Phytoseiidae, Raphignathidae, Rhagidiidae, Stigmaeidae and Uropodidae. The remaining species belonging to families of diverse or inadequately known feeding habits, which are here categorized as "generalists" (Acaridae, Ameroseiidae, Aphelacaridae, Cosmochthoniidae, Ctenacaridae, Epilohmanniidae, Galumnidae, Glycyphagidae, Haplochthoniidae, Haplozetidae, Lohmanniidae, Oppiidae, Oribatulidae, Pygmephoridae, Siteroptidae, Tarsonemidae and Tydeidae). Generalists are thought to comprise detritivorous, aligivorous, fungivorous, bacteriophagous and pollenophagous organisms (Walter and O'Dowd, 1995).

\section{List of mites recorded}

\section{ORDER ACARIDIDA}

Family Acaridae Ewing \& Nesbitt

1. Acarus siro L. Host plant: F. nitida, $R$. ragia.

2. Rhizoglyphus callae Oudemans Host plant: T. majus, D. nutans.

3. $R$ robini Claparède Host plant: A. saligna.

4. Tyrophagus brevicrinatus Robertson 
Host plant: $M$. viridis, $C$. pyramidalis, B. spectabilis, Begonia sp., P. alba, Q. indica, $S$. reginae, $A$. vasica, $C$. variegatum.

5. T. longior (Gervais)

Host plant: $O$. basilicum, $C$. asiaticum.

6. T. perniciosus Zachvatkin

Host plant: $C$. sempervirens, $F$. nitida, $H$. sabderiffa, $P$. tuberosa, C. splendens.

7. T. putrescentiae (Schrank)

Host plant: $A$. graveolens, $P$. sativum, $C$. fruticosa, A. umbellatus, $G$. jamesonii, $M$. Periwinkle, $C$. pyramidalis, C. equsetifolia, $A$. excelsa, $D$. fragrans, $P$. pubescens, $C$. sempervirens, $F$. nitida, $F$. benghalensis, $J$. sambac, $R$. hybrida, $S$. podophyllum, Begonia sp., A. wilkesiana, $P$. canariensis, $P$. dactylifera, $C$. mitis, $R$. ragia, $A$. vasica, $C$. variegatum, $C$. splendens, $D$. thunbergiana.

8. T. similis Volgin

Host plant: A. maritima, L. nobilis , $P$. somniferum, $M$. incana, $H$. annus, $V$. tricolor, $A$. majus, $C$. pyramidalis, $K$. panniculata, $A$. paniculata, A. saligna, B. hookeru, $F$. nitida, $Y$. gloriosa, L. indica, Q. indica, W. filifera, A. vasica, C. splendens, D. thunbergiana.

9. T. tropicus Robertson

Host plant: $S$. leucantha, $F$. nitida, $F$. benghalensis, $E$. pulcherrima, $R$. ragia, $D$. thunbergiana.

\section{Family Glycyphagidae Berlese}

1. Austroglycyphagus geniculatus (Vitzthum)

Host plant: L. camara.

2. Glycyphagus domesticus (De Geer)

Host plant: $C$. pyramidalis, $C$. sempervirens, $L$. indica, I. palmata, Begonia sp., W. filifera, C. variegatum, $D$. thunbergiana.

\section{ORDER ACTINEDIDA}

\section{Family Bdellidae Dugès}

1. Cyta latirostris (Hermann)

Host plant: $A$. majus, $R$. communis, $S$. officinalis, $E$. globulus, $F$. vulgare, $A$. indica, $P$. somniferum, $A$. graveolins, $M$. viridis, $O$. marjorana, $P$. sativum, $H$. annus, $V$. tricolor, A. majus, $C$. fruticosa, $A$. umbellatus, G. jamesonii, M. Periwinkle, M. genistifolia, $P$. tobira, $F$. sycamorus, $E$. variegata, $M$. deliciosa, $D$. nutans, $D$. regia, $C$. sempervirens, $A$. saligna, $B$. hookeru, $F$. benghalensis, $Y$. gloriosa, $N$. oleander, C. asiaticum, $H$. sabderiffa, $R$. hybrida, L. indica, $T$. stans, $B$. spectabilis, S. podophyllum, J. grandiflorum, Begonia sp.,
$A$. wilkesiana, $P$. alba, $P$. tuberosa, $Q$. indica, $M$. exotica, $P$. dactylifera, $\boldsymbol{R}$ ragia, $A$. vasica, $C$. variegatum, $C$. splendens, $D$. thunbergiana.

2. Neomolgus aegyptiacus Soliman

Host plant: $M$ viridis, $O$. basilicum, $S$. leucantha, $C$. pyramidalis, $S$. nilotica, $P$. zonale, $C$. mitis, $R$ ragia.

3. Spimibdella bifurcata Atyeo

Host plant: C. papaya, $M$. chamomilla, $C$. ambrosioide, $L$. nobilis, $C$. sativum, $C$. officinalis, $M$. viridis, $P$. pubescens, $F$. nitida, $P$. alba, $W$. filifera, $R$ ragia, C. variegatum.

\section{Family Caligonellidae Grandjean}

1. Caligonella humilis Grandjean

Host plant: C. pyramidalis.

2. Molothrognathus minutus Soliman

Host plant: $P$. canariensis.

\section{Family Camerobiidae Southcott}

Camerobia gonzali (Zaher \& Gomaa)

Host plant: $D$. regia, $H$. sabderiffa, $I$. palmata, B. spectabilis.

\section{Family Cheyletidae Leach}

1. Acaropsella kulagini (Rohdendorf)

Host plant: $F$. sycamorus, $M$. alba, $M$. nigra, $D$. nutans, D. thunbergiana.

2. A. notchi Gomaa \& Hassan

Host plant: $F$. benghalensis, $A$. vasica, D. thunbergiana.

3. Acaropsellina (Acaropsis) sollers (Rohdendorf) Host plant: $C$. aurantium.

4. Cheletogenes ornatus (Canestrini \& Fanzago) Host plant: E. variegata.

5. Cheletomorpha lepidopterorum (Shaw)

Host plant: $A$. graveolins, $P$. pubescens, D. thunbergiana.

6. Chelotominus (Hemicheyletia) bakeri (Ehara) Host plant: $R$. communis, $L$. nobilis, $C$. sativum, A. graveolens, $O$. basilicum, $O$. marjorana, A. umbellatus, S. leucantha, M. Periwinkle, $C$. nodosa, $S$. nilotica, $C$. sempervirens, B. hookeru, F. nitida, $R$. hybrida, E. pulcherrima, L. indica, $T$. stans, $S$. podophyllum, J. grandiflorum, Begonia sp., A. wilkesiana, $P$. alba, $P$. tuberosa, $M$. exotica, $P$. canariensis, $P$. dactylifera, $C$. mitis, $R$. ragia, $S$. reginae, $C$. variegatum, $C$. splendens, $D$. thunbergiana.

7. C. congensis (Cunliffe)

Host plant: $C$. variegatum.

8. C. volgini (Cunliffe)

Host plant: $S$. leucantha, $F$. nitida.

9. C. wellsi (Baker)

Host plant: $F$. nitida.

10. Cheyletus aversor Rohdendorf 
Host plant: W. filifera.

11. Ch. cacahuamilpensis Baker Host plant: E. globulus, S. nilotica, A. vasica.

12. Mexecheles cunliffei De Leon Host plant: L. nobilis.

\section{Family Cunaxidae Thor}

1. Armascirus anastasi Lindquist Host plant: A. wilkesiana.

2. Coleoscirus buartus Den Heyer Host plant: C. mitis.

3. Cunaxa capreolus (Berlese) Host plant: $H$. helix, C. sativum, A. graveolens, I. palmata, $P$. alba, $C$. splendens, D. thunbergiana.

4. Neocunaxoides andrei (Baker \& Hoffmann) Host plant: $C$. officinalis, $M$. viridis, $O$. basilicum, O. marjorana, M. Periwinkle, C. pyramidalis, $M$. deliciosa, $S$ actinophylla, $D$. fragrans, $D$. nutans, $S$. babylanica, A. saligna, B. hookeru, $F$. nitida, Y. gloriosa, $P$. zonale, $R$. hybrida, S. podophyllum, Begonia sp., A. marginta, $P$. alba, $Q$. indica, $M$. exotica, $C$. revoluta, $\boldsymbol{P}$. dactylifera, $W$. filifera, $C$. mitis, $R$. ragia, $S$ reginae, $A$. vasica, $C$. variegatum, C. splendens, D. thunbergiana.

5. N. rykei Den Heyer

Host plant: $M$. viridis, $O$. marjorana, C. fruticosa, $S$. leucantha, $R$. hybrida, S. podophyllum, A. marginta, A. wilkesiana, P. tuberosa, W. filifera, C. mitis, A. vasica, C. variegatum, C. splendens.

6. Pulaeus americanus (Baker \& Hoffmann) Host plant: $P$. pubescens, $C$. mitis.

7. P. glebulentus Den Heyer

Host plant: $F$. benghalensis, $C$. mitis, $A$. vasica, $D$. thunbergiana.

8. P. subterraneus (Berlese)

Host plant: P. pubescens, A. wilkesiana.

\section{Family Eupalopsellidae Willmann}

1. Eupalopsellus olearius Zaher \& Gomaa Host plant: C. variegatum.

2. Saniosulus nudus Summers

Host plant: L. nobilis, C. pyramidalis, P. alba, $R$. ragia.

\section{Family Eupodidae Koch}

1. Egypteupodes strandmanni Abou-Awad

Host plant: D. regia, J. grandiflorum, A. wilkesiana, $P$. tuberosa, $M$. exotica, D. thunbergiana.

2. Eupodes momeni Abou-Awad

Host plant: M. Periwinkle, M. nigra, S. nilotica, T. stans, $S$. podophyllum, $P$. tuberosa, C. variegatum.
3. E. niloticus Abou Awad \& El-Bagoury Host plant: $L$. nobilis, $P$. sativum, D. regia.

4. E. temperatus Shiba

Host plant: $M$. chamomilla, $S$. officinalis, $F$. vulgare, $P$. somniferum, $C$. sativum, A. graveolens, A. graveolins, C. officinalis, $M$. viridis, $O$. basilicum, $O$. marjorana, $P$. hybrida, $M$. incana, $C$. fruticosa, A. umbellatus, G. jamesonii, S. leucantha, M. Periwinkle, C. pyramidalis, $P$. tobira, $P$. halepensis, A. saligna, $C$. asiaticum, $J$. sambac, $R$. hybrida, J. grandiflorum, Begonia sp., A. marginta, $P$. alba, C. revoluta, $W$. filifera, $C$. mitis, $S$. reginae, $A$. vasica, $C$. variegatum, C. splendens, D. thunbergiana.

5. E. voxencollinus Sig Thor

Host plant: A. umbellatus, $P$. tobira, E. pulcherrima, T. stans, $W$. filifera.

\section{Family Pygmephoridae Cross}

Pediculaster gallinae Zaher \& Kandeel

Host plant: $W$. filifera, $S$. reginae.

\section{Family Raphignathidae Kramer}

1. Raphignathus bakeri Zaher \& Gomaa Host plant: M. Periwinkle, A. wilkesiana.

2. $R$. collegiatus Atyeo, Baker \& Crossley Host plant: L. indica.

3. R. ehari Zaher \& Gomaa Host plant: M. Periwinkle, F. nitida, P. alba.

4. $R$. evansi Zaher \& Gomaa Host plant: $A$. vasica.

5. R. gracilis (Rack) Host plant: $F$. nitida.

6. $R$. niloticus Rakha \& Mohamed Host plant: $H$. helix, $M$. viridis, $M$. nigra, $D$. nutans, D. regia, $S$. babylanica, $R$. hybrida, L. indica, I. palmata, B. spectabilis, L. camara, $J$. grandiflorum, Begonia sp., A. wilkesiana, $P$. dactylifera, $S$. reginae, $A$. vasica, C. variegatum, D. thunbergiana.

\section{Family Rhagidiidae Oudemans}

1. Robustocheles mucronata (Willmann) Host plant: C. asiaticum.

2. Shibaia shereefi Abou-Awad Host plant: G. jamesonii, T. stans, C. mitis, C. splendens.

\section{Family Siteroptidae Mahunka}

Siteroptes manurei Soliman \& Kandeel Host plant: $P$. pubescens.

\section{Family Stigmaeidae Oudemans}

1. Agistemus exsertus Gonzalez Host plant: $O$. marjorana, $C$. pyramidalis, $D$. nutans, $F$. nitida, $H$. sabderiffa, $R$. hybrida, 
I. palmata, J. grandiflorum, A. marginta, $P$. alba, $P$. tuberosa, $Q$. indica, $S$. reginae, A. vasica, C. variegatum, C. splendens, D. thunbergiana.

2. A. vulgaris Soliman \& Gomaa Host plant: $O$. marjorana.

3. Stigmaeus africanus Soliman \& Gomaa Host plant: $A$. wilkesiana, $M$. exotica, $R$. ragia, D. thunbergiana.

\section{Family Tarsonemidae Canestrini \& Fanzago}

1. Steneotarsonemus hatzinikolisi Emmanouel Host plant: $S$. officinalis, A. graveolens, $B$. hookeru, R. hybrida, D. thunbergiana.

2. Tarsonemus meyerus Soliman \& Kandeel Host plant: L. nobilis, C. sativum, C. officinalis, $O$. basilicum, $M$. genistifolia, $K$. panniculata, $S$. nilotica, C. asiaticum, Begonia sp., P. tuberosa, $M$. exotica, $C$. revoluta, $P$. canariensis, $R$. ragia, $S$. reginae, $A$. vasica, C. variegatum, C. splendens.

\section{Family Tenuipalpidae Berlese}

1. Aegyptobia salixi Zaher \& Yousef Host plant: $M$. viridis.

2. A. sayedi Yousef Host plant: $M$. viridis, $R$. hybrida

3. Brevipalpus obovatus Donnadieu

Host plant: $H$. helix, M. piperita, C. officinalis, $M$. viridis, $O$. basilicum, $O$. marjorana, $G$. jamesonii, $S$. babylanica, $H$. sabderiffa, E. pulcherrima, I. palmata, Begonia sp., P. alba, $R$. ragia, $S$ reginae, $C$. splendens.

4. B. olearius Sayed Host plant: $P$. alba.

5. Cenopalpus pulcher (Canestrini \& Fanzago) Host plant: A. rosae, Begonia sp.

\section{Family Tetranychidae Donnadieu}

\section{Bryobia cristata (Duges)}

Host plant: C. aurantium, A. majus, $R$ officinale, M. chamomilla, S. officinalis, $P$. somniferum, A. graveolins, C. officinalis, $P$. sativum, $M$. incana, $A$. saligna, $W$. filifera, D. thunbergiana.

2. B. praetiosa Koch Host plant: G. jamesonii, T. distichum.

3. Eotetranychus zacheri Zaher, Gomaa \& El-Enany Host plant: $M$. piperita, $F$. nitida, $N$. oleander, A. vasica, D. thumbergiana.

4. Eutetranychus africanus (Tücker) Host plant: $P$. alba.

5. E. pyri Attiah Host plant: $P$. alba.

6. Oligonychus afrasiasticus (McGregor) Host plant: $F$. nitida.
7. O. mangiferus (Rahman \& Sapra) Host plant: $P$. alba.

8. O. punicae (Hirst) Host plant: $O$. marjorana, $R$. hybrida, $P$. alba.

9. O. sayedi Zaher, Gomaa \& El-Enany Host plant: $F$. nitida, $R$. hybrida.

10. Panonychus ulmi (Koch)

Host plant: $M$. viridis, $S$. reginae, D. thunbergiana.

11. Tetranychina harti (Ewing)

Host plant: $C$. papaya, $C$. officinalis, $M$. viridis, A. maritimum, $S$. leucantha, $P$. tomentosa, $C$. citrinus, $C$. nodosa, $F$. nitida, $R$. hybrida, Begonia sp., A. wilkesiana, W. filifera, $R$. ragia, S. reginae.

12. Tetranychus cucurbitacearum (Sayed)

Host plant: C. papaya, A. majus, R. communis, A. maritima, $F$. vulgare, $P$. somniferum, C. sativum, A. graveolens, A. graveolins, $C$. officinalis, $M$. viridis, $O$. marjorana, $P$. sativum, $H$. annus, $V$. tricolor, $A$. umbellatus, M. Periwinkle, C. pyramidalis, T. distichum, A. excelsa, $P$. pubescens, A. saligna, $N$. oleander, $J$. sambac, $R$. hybrida, $P$. alba, $P$. tuberosa, $M$. exotica, $P$. canariensis, $W$. filifera, C. mitis, A. vasica, C. variegatum, C. splendens.

13. T. neocalidonicus André

Host plant: E. globulus, C. officinalis, M. Periwinkle, $R$. hybrida.

14. T. urticae Koch

Host plant: C. papaya, C. ambrosioide, L. nobilis, $M$. viridis, $O$. basilicum, O. marjorana, $P$. sativum, T. majus, $A$. rosae, A. majus, G. jamesonii, M. alba, B. hookeru, J. sambac, $R$. hybrida, L. camara, $J$. grandiflorum, Begonia sp., A. wilkesiana, P. alba, P. tuberosa, $C$. splendens, D. thunbergiana.

\section{Family Tydeidae Kramer}

1. Paralorryia aegyptiaca Rasmy \& El-Bagoury Host plant: $M$. exotica, D. thunbergiana.

2. P. bakeri Zaher \& El-Bagoury Host plant: $P$. sativum, $A$. saligna, $W$. filifera.

3. P. ferula (Baker) Host plant: $M$. exotica.

4. P. gizai El-Bagoury

Host plant: $M$. viridis, $O$. basilicum, O. marjorana, $P$. hybrida, $V$. tricolor, $M$. genistifolia, $P$. pubescens, $F$. nitida, $F$. benghalensis, B. spectabilis, Begonia sp., $A$. marginta, $A$. wilkesiana, $P$. alba, $C$. revoluta, $P$. dactylifera, $C$. mitis, $S$. reginae, $C$. variegatum, $C$. splendens.

5. P. mali (Oudemans) 
Host plant: $A$. excelsa, $C$. sempervirens, A. vasica, D. thunbergiana.

6. P. woolleyi Baker

Host plant: $A$. indica, $G$. jamesonii, $C$. splendens.

7. P. zaheri Baker

Host plant: $D$. thunbergiana.

8. Paratriophtydeus plummeri (Baker)

Host plant: $L$. nobilis, S. leucantha, $H$. sabderiffa, $R$. ragia, $C$. splendens.

9. Pronematus rykeri Meyer \& Rodriguez Host plant: $C$. officinalis.

10. P. ubiquitus (McGregor) Host plant: $B$. hookeru.

11. Triophtydeus vitis El-Bagoury Host plant: $R$. hybrida, I. palmata.

12. Tydeus californicus (Banks)

Host plant: $P$. sativum, $E$. variegata, H. sabderiffa, L. indica, P. alba, A. vasica.

13. T. costensis Baker

Host plant: $P$. somniferum, A. graveolens, O. marjorana, M. Periwinkle, S. babylanica, R. hybrida, A. vasica, C. splendens.

14. T. kochi Oudemans Host plant: C. mitis.

15. T. mississippiensis Baker Host plant: $S$. officinalis, $Q$. indica.

16. $T$. oregonensis Baker

Host plant: C. asiaticum, I. palmata, A. vasica, C. splendens.

17. T. schusteri André \& Naudo

Host plant: Begonia sp.

\section{ORDER GAMASIDA}

\section{Family Ameroseiidae Evans}

1. Kleemannia kosii El-Badry, Nasr \& Hafez Host plant: $A$. wilkesiana, D. thunbergiana.

2. K. plumosus (Oudemans)

Host plant: $L$. nobilis, $A$. indica, $A$. graveolens, A. graveolins, $M$. viridis, $P$. sativum, $P$. pubescens, C. sempervirens, B. hookeru, $F$. nitida, $R$. hybrida , $T$. stans, $L$. camara, $M$. exotica, $S$. reginae, $A$. vasica, D. thunbergiana.

3. K. zaheri El-Badry, Nasr \& Hafez Host plant: $A$. vasica.

\section{Family Ascidae Voigts \& Oudemans}

1. Blattisocius tarsalis (Berlease)

Host plant: $C$. variegatum.

2. Gamasellodes bicolor (Berlease)

Host plant: $P$. sativum.

3. Lasioseius bispinosus Evans Host plant: L. nobilis.

4. L. lindquisti Nasr \& Abou-Awad

Host plant: $T$. stans, $C$. mitis, $R$. ragia,
C. variegatum, $C$. splendens.

5. L. peritremus Nasr \& Abou-Awad

Host plant: $O$. marjorana.

6. L. sewai Nasr \& Abou-Awad

Host plant: $M$. viridis, $C$. pyramidalis, R. hybrida, E. pulcherrima, C. mitis, C. variegatum.

7. Proctolaelaps aegyptiaca Nasr Host plant: $F$. vulgare, $C$. sativum, $S$. babylanica, Begonia sp. ,S. reginae, $C$. splendens.

8. $P$. orientalis $\mathrm{Nasr}$

Host plant: A. graveolens, $A$. graveolins, $C$. officinalis, $M$. viridis, $O$. marjorana, C. pyramidalis, $A$. excelsa, $F$. nitida, $F$. benghalensis, E. pulcherrima, S. podophyllum, $J$. grandiflorum, Begonia sp., $Q$. indica, C. revoluta.

9. P. pygmaeus (Müller)

Host plant: $M$. viridis, $O$. basilicum, $O$. marjorana, $R$. hybrida, A. marginta, $M$. exotica, $W$. filifera, $S$. reginae, $A$. vasica, C. splendens.

10.P. striatus Afifi, Hassan \& El-Bishlawy Host plant: $A$. graveolins.

\section{Family Digamasellidae Evans}

1. Dendrolaelaps aegypticus Metwally \& Mersal Host plant: $C$. pyramidalis.

2. D. zaheri Metwally \& Mersal

Host plant: $D$. fragrans.

Family Laelapidae Berlese

1. Androlaelaps aegypticus Hafez, El-Badry \& Nasr Host plant: Begonia sp., C. splendens, D. thunbergiana.

2. A. casalis (Berlese)

Host plant: $P$. somniferum, $M$. Periwinkle, $D$. regia, $A$. saligna, $F$. nitida, $R$. hybrida, $S$. podophyllum, J. grandiflorum, C. revoluta, C. splendens, D. thunbergiana.

3. A. reticulatus Hafez, El-Badry \& Nasr Host plant: $P$. sativum, $G$. jamesonii, $J$. grandiflorum, $S$. reginae, $C$. splendens, D. thunbergiana.

4. A. zaheri Hafez, El-Badry \& Nasr Host plant: $M$. exotica.

5. Cosmolaelaps keni Hafez, El-Badry \& Nasr Host plant: $M$. viridis, $M$. Periwinkle, S. actinophylla, $A$. wilkesiana.

6. C. longus Hafez, El-Badry \& Nasr Host plant: $P$. tuberosa.

7. Hypoaspis gergus Hafez, El-Badry \& Nasr Host plant: $O$. basilicum, $C$. variegatum.

8. H. orientalis Hafez, El-Badry \& Nasr Host plant: $S$. reginae.

9. Laelaspis astronomicus (Koch) 
Host plant: M. Periwinkle, C. pyramidalis, Begonia sp., A. wilkesiana, P. alba, P. tuberosa, $C$. variegatum, $C$. splendens, $D$. thunbergiana.

10. Ololaelaps bregetovae Shereef \& Soliman

Host plant: $A$. graveolens, $C$. officinalis, $M$. viridis, $C$. sempervirens, $P$. alba, $C$. mitis, R. ragia.

11. Stratiolaelaps miles (Berlese)

Host plant: L. camara.

\section{Family Macrochelidae Vitzthum}

1. Macrocheles punctata Hafez, El-Badry \& Nasr Host plant: A. majus, $M$. viridis, $S$. leucantha, P. zonale.

2. M. sembelawanii Hafez, El-Badry \& Nasr Host plant: $R$. hybrida.

\section{Family Ologamasidae Ryke}

Gamasiphis pulchellus (Berlease)

Host plant: $M$. viridis, O. marjorana, $M$. incana, $S$. leucantha, $M$. Periwinkle, A. paniculata, $S$. babylanica, $A$. saligna, B. hookeru, $F$. nitida, $Y$. gloriosa, R. hybrida , E. pulcherrima, $S$. podophyllum, Begonia sp., A. wilkesiana, $P$. tuberosa, M. exotica, C. revoluta, $R$. ragia, $S$. reginae, $A$. vasica, $C$. splendens, D. thunbergiana.

\section{Family Pachylaelapidae Berlese}

Pachylaelaps reticulatus Hafez \& Nasr

Host plant: $O$. marjorana, $V$. tricolor, $R$. hybrida, Begonia sp.

\section{Family Parasitidae Oudemans}

\section{Eugamasus cavernicola Trägårdh} Host plant: $R$. ragia.

2. Gamasodes spiniger (Trägårdh)

Host plant: $F$. benghalensis, Begonia sp., $R$. ragia, $C$. splendens, $D$. thunbergiana.

3. Parasitellus fucorum (De Geer) Host plant: $R$. ragia.

4. Parasitus beta Oudemans \& Voigts

Host plant: $M$. viridis, $S$. leucantha, $B$. spectabilis, $W$. filifera, $R$. ragia, C. variegatum, $C$. splendens.

5. $P$. consanguineus Oudemans $\&$ Voigts Host plant: A. marginta, $S$. reginae.

6. $P$. fimetorum (Berlese)

Host plant: $O$. basilicum, $S$. babylanica, $R$. hybrida, $J$. grandiflorum, $R$. ragia, C. variegatum.

7. P. hyalinus (Willmann) Host plant: $P$. alba, $R$ ragia.

8. Porrhostaspis lunulata Müller Host plant: $R$. ragia.

9. Vulgarogamasus remberti (Oudemans) Host plant: A. majus, R. hybrida.

\section{Family Phytoseiidae Berlese}

1. Euseius plumerii Abo-Shnaf \& Romeih sp. $\mathrm{n}$.

Host plant: L. nobilis, $O$. marjorana, $A$. rosae, $S$. leucantha, $C$. pyramidalis, A. paniculata, $B$. hookeru, $F$. nitida, $H$. sabderiffa, $R$. hybrida, I. palmata, L. camara, Begonia sp., A. marginta, A. wilkesiana, $P$. alba, Q. indica, A. vasica, $C$. variegatum, $C$. splendens.

2. Neoseiulella (Typhlodromus) schusteri (Yousef \& El-Brollosy)

Host plant: $M$. viridis.

3. Neoseiulus barkeri (Hughes) Synonym Amblyseius cydnodactylon Shehata \& Zaher

Host plant: $M$. piperita, A. maritima, A. graveolins, $C$. officinalis, $M$. viridis, $O$. basilicum, $O$. marjorana, $P$. sativum, $V$. tricolor, $S$. leucantha, $M$. Periwinkle, $C$. pyramidalis, $A$. paniculata, $S$. nilotica, $F$. nitida, $R$. hybrida, E. pulcherrima, Begonia sp., $A$. marginta, $P$. alba, $Q$. indica, $W$. filifera, $C$. mitis, $R$. ragia, $S$. reginae, $A$. vasica, C. variegatum, $D$. thunbergiana.

4. N. cucumeris (Oudemans)

Host plant: A. marginta, $P$. alba.

5. $N$. enab El-Badry

Host plant: $C$. ambrosioide, $F$. nitida.

6. N. longispinosus (Evans)

Host plant: $N$. oleander, $L$. indica, C. splendens.

7. Typhlodromips. swirskii Athias-Henriot

Host plant: $R$. hybrida.

8. Typhlodromus balanites El-Badry

Host plant: B. spectabilis.

9. $T$. pyri Schueten

Host plant: $A$. marginta

10.T. siwa El-Badry

Host plant: $M$. chamomilla, $F$. vulgare, $A$. graveolens, $M$. viridis, $O$. basilicum,

$O$. marjorana, $C$. sempervirens, $F$. nitida, $J$ sambac, J. grandiflorum, C. revoluta.

11.T. tetramedius Zaher \& Shehata

Host plant: A. graveolins.

\section{Family Uropodidae Berlese}

1. Trichouropoda patavina (Canestrini)

Host plant: $A$. indica.

2. Uroobovella krantzi Zaher \& Afifi

Host plant: $F$. benghalensis, $C$. mitis.

\section{ORDER ORIBATIDA}

Family Aphelacaridae Grandjean

Aphelacarus acarinus (Berlese)

Host plant: $P$. dactylifera.

Family Cosmochthoniidae Grandjean

Cosmochthonius lanatus (Michael) 
Host plant: N. oleander, J. grandiflorum, R. ragia.

\section{Family Ctenacaridae Grandjean}

Ctenacarus araneola (Grandjean)

Host plant: C. ambrosioide, M. alba, M. nigra, L. indica, $W$. filifera.

\section{Family Epilohmanniidae Oudemans}

Epilohmannia cylindrica Berlese

Host plant: $O$. marjorana, A. umbellatus, $P$. tobira, $S$. actinophylla, $P$. zonale, $A$. marginta, C. mitis, $A$. vasica, $C$. variegatum, $C$. splendens.

\section{Family Galumnidae Jacot}

1. Galumna flabillifera Hammer

Host plant: $O$. basilicum, $F$. nitida, $M$. exotica, D. thunbergiana.

2. Pilogalumna sp.

Host plant: $M$. viridis, $O$. basilicum, $O$. marjorana, A. majus, $S$. leucantha, $F$. benghalensis, $P$. zonale, $R$. hybrida, $A$. wilkesiana, $Q$. indica, $S$. reginae, $A$. vasica, D. thunbergiana.

\section{Family Haplochthoniidae Hammen}

Haplochthonius africanus Yousef \& Nasr

Host plant: E. globulus, $L$. indica, $J$. grandiflorum, $P$. dactylifera, $D$. thunbergiana.

\section{Family Haplozetidae Grandjean}

1. Perxylobates mahunkai Bayoumi Host plant: P. sativum.

2. Rostrozetes aegypticus (Yousef \& Nasr) Host plant: $A$. saligna, $B$. spectabilis, A. wilkesiana.

3. R. africanus (Yousef \& Nasr) Host plant: Begonia sp.

4. R. citri Nasr \& Hassan Host plant: $R$. hybrida, E. pulcherrima.

5. Xylobates souchnaiensis Abdel-Hamid

Host plant: $A$. graveolens, $M$. viridis, $O$. marjorana, $C$. pyramidalis, $P$. halepensis, $S$. babylanica, $B$. hookeru, $R$. hybrida, $A$. marginta, $A$. wilkesiana, $Q$. indica, $R$. ragia, $S$. reginae, $C$. variegatum, $C$. splendens.

\section{Family Lohmanniidae Berlese}

1. Lohmannia egypticus (El-Badry \& Nasr)

Host plant: $M$. viridis, $O$. marjorana, $S$. leucantha, $C$. pyramidalis, S. actinophylla, $B$. hookeru, $F$. nitida, A. marginta, $Q$. indica, $C$. revoluta, $W$. filifera, $C$. mitis, $S$. reginae, C. variegatum, C. splendens.

2. Papillacarus aciculatus Kunst Host plant: Begonia sp.

\section{Family Oppiidae Grandjean}

1. Oppia bayoumii Shereef \& Zaher

Host plant: A. majus, S. reginae.

2. O. concolor Koch

Host plant: $M$. viridis, $O$. basilicum, $O$. marjorana, A. maritimum, C. pyramidalis, O. marjorana, A. maritimum, C. pyramidalis,

$D$. regia, $F$. nitida, $F$. benghalensis, $Y$. gloriosa, $R$. hybrida, S. podophyllum, A. marginta, $A$. wilkesiana, $Q$. indica, $M$. exotica, C. mitis,

$S$. reginae, $C$. splendens, D. thunbergiana.

3. O. sticta Popp

Host plant: $A$. indica, $P$. tuberosa

\section{Family Oribatulidae Thor}

\section{Scheloribates laevigatus (Koch)}

Host plant: $M$. chamomilla, $A$. indica, $C$. officinalis, A. majus, G. jamesonii, D. nutans, $S$. podophyllum, $S$. reginae, $C$. variegatum, D. thunbergiana.

2. $S$. zaheri Yousef \& Nasr

Host plant: $A$. majus, $R$. aculeatus, $M$. viridis, $O$. basilicum, $O$. marjorana, $C$. fruticosa, A. umbellatus, M. Periwinkle, C. pyramidalis, $T$. distichum, $D$. fragrans, $B$. hookeru, $F$. nitida, $J$. sambac, $R$. hybrida, $S$. podophyllum, Begonia sp., A. marginta, $P$. tuberosa, $Q$. indica, $C$. revoluta, $P$. canariensis, $P$. dactylifera, $W$. filifera, $C$. mitis, $R$. ragia, $S$. reginae, $A$. vasica, $C$. variegatum, $C$. splendens, D. thunbergiana.

3. Zygoribatula sayedi El-Badry \& Nasr

Host plant: $C$. sativum, $A$. graveolins, C. officinalis, $M$ viridis, $O$. basilicum, $P$. sativum, $R$. hybrida, $P$. tuberosa.

4. Z. tritici El-Badry \& Nasr,

Host plant: $F$. vulgare, $C$. sativum, A. graveolens, $P$. sativum, $A$. maritimum, $C$. pyramidalis, $F$. nitida, $R$. hybrida, $M$. exotica.

These findings are likewise those presented by Hanna et al. (1981) who listed some of these previous species among the collected mites of 46 ornamental and medicinal plants at Giza and Qualyoubia Governorates, which averaged 29 mite species. In addition, some of these species were recorded by Hassan et at. (1986) who collected mites inhabiting desert plants in Sinai Peninsula regions and new reclaimed lands and sorted 110 mite species belonging to 68 genera and 34 families.

Among the collected species, one species belongs to genus Euseius, E. plumerii sp. n. of family Phytoseiidae. Some new phytoseiid species of genus Euseius, have been observed by Qayyum et al. 
(2004); Bashir et al. (2006) and Bayan \& Merheb (2006). Meanwhile, 24 species are firstly recorded on plants or in soil belongs to several families; seven species of the family Acaridae: $A$. siro, $R$. callae, $T$. brevicrinatus, $T$. longior, $T$. perniciosus, T. tropicus and T. similis. Six species of the family Parasitidae: E. cavernicola, G. spiniger, P. fucorum, $P$. hyalinus, $P$. lunulata and $V$. remberti. Five species to each of the families; Cunaxidae: A. anastosi, C. buartus, $P$. americanus, $P$. glebulentus and $P$. subterraneus; and the family Cheyletidae: A. kulagini, A. sollers, C. volgini, $C$. wellsi and $M$. cunliffei. Koç (1998) recorded the cheyletid mite, $A$. volgini from Turkey's fauna. Dogan and Ayyildiz (2004) recorded the two cheyletid mites, C. wellsi and Hypopicheyla elongata Volgin from Turkey. Moreover, one species of the family Tarsonemidae, $S$. hatzinikolisi has been recorded for the first time.

\section{REFERENCES}

Amro, M. A. M., 2007. Analytical review of the main arthropod pests and natural enemies associated with $P$. dactylifera trees in Egypt. $1^{\text {st }}$ international conference of $P$. dactylifera, integrated crop management of $P$. dactylifera and its impacts for producing clean and safety dates, September 2007 Giza, Egypt: 7.

Bashir, M. H.; Afzal, M. and Ahmad, J. N., 2006. Description of two new phytoseiid mites of genera Neoseiulus and Euseius from district Rahim Yar Khan. Pak. Entomol., 28 (2): 57-62.

Bayan, A. and Merheb, B., 2006. Descriptions of two new phytoseiid mites (Acari: Mesostigmata: Gamasina) found in a grapevine orchard from Lebanon. Sys. Appl. Acarol., 11: 51-56.

Cui, Y.B.; Li, C.P.; Wang, J.; Yang, Q.G.; Tian, Y. and Tao, L., 2005. Astigmatid mites infesting Chinese medicinal herbs. Acarina, 13 (2): 203208.

Doĝan, S. and Ayyildiz, N. 2004. The first records of two cheyletid mites from Turkey: Chelotominus (Hemicheyletia) wellsi (Baker, 1949) and Hypopicheyla elongata Volgin, 1969 (Acari: Cheyletidae). Zootaxa, 583: 1-4.

Fan, Q. H.; Zhang, Y. X. and Liu, Q. Y., 2000. Raphignathoid mites on bamboo from Fujian, China (Acari: Prostigmata). Sys. Appl. Acarol., 4: 49-68.

Friend, J. A.; Singh, R. P. (ed.) and Saxena, R. C., 1999. Pest and diseases of $A$. indica (Azadirachta indica A. Juss.) in Australia and associated biota. Azadirachta indica A. Juss, 2133. A indica Peace, P.O. Box 362, Edge Hill, Qld 4870, Australia.
Gallo, D.; Nakano, O.; Silveira-Neto, S.; Carvalho, R. P. L.; Baptista, G. C.; Berti-Filho, E.; Parra, J. R. P.; Zucchi, R. A.; Alves, S. B.; Vendramim, J. D.; Marchini, L. C.; Lopes, J. R. S. and Omoto, C., 2002. Entomologia Agrícola. Piracicaba: FEALQ: 920.

Hanna, M. A. Shereef; G. M. and Megali, Magda K., 1981. Mites associated with ornamental and medicinal plants in Egypt. Bull. Soc. Entomol. Egypte, 63: 43-47.

Hassan, M. F.; Afifi, A. M. and Nawar, M. S., 1986. Mites inhabiting plants and soil in Sinai and newly reclaimed lands. Bull. Soc. Entomol. Egypte, 66: 211-225.

Jesse, L. C.; Moloney, K. A. and Obrycki, J. J., 2006. Abundance of arthropods on the branch tips of the invasive plant, Rosa multiflora Thunb. (Rosaceae). Weed Biol. Manag., 6: 204-211.

Karlik, J. F.; Goodell, P. B. and Osteen, G. W., 1995. Improved mite sampling may reduce acaricide use in roses. California Agriculture, 49(1-4): 38-40.

Koç, K., 1998. A new record of Acaropsella volgini (Acari: Prostigmata, Cheyletidae) for the fauna of Turkey. Tr. J. Zoology, 22: 195-197.

Lawson-Balagbo, L. M.; Gondim, M. G. C. Jr; Moraes, G. J. de; Hanna, R. and Schausberger, P., 2008. Exploration of the acarine fauna on coconut palm in Brazil with emphasis on Aceria guerreronis (Acari: Eriophyidae) and its natural enemies. Bull. Entomol. Res., 98: 83-96.

Mohamed, M. I. and Hassan, M. F., 1984. Survey and biological studies on mites inhabiting fruit and ornamental trees in the Netherlands. Bull. Fac. Agric., Univ. Cairo, 35 (1): 641-652.

Moraes, G. J. de; Lopes, P. C. and Fernando, L. C. P., 2004. Phytoseiid mites (Acari: Phytoseiidae) of coconut growing areas in Sri Lanka, with descriptions of three new species. J. Acarol. Soc. Jpn., 13 (2): 141-160.

Palmer, W. A. and Pullen, K. R., 1995. The

phytophagous arthropods associated with $L$. camara camara, L. hirsuta, L. urticifolia, and $L$. urticoides (Verbenaceae) in North America. Biol. Cont., 5 (1): 54-72.

Qayyum, S.; Pervez, I. and Afzal, M., 2004. New species of the genus Euseius (Acarina: Phytoseiidae) from northern areas of Pakistan. Pak. J. Agri. Sci., 4l(3-4): 146-151.

Rosenheim, J. and Fournier, V., 2007. Additivity versus interactions in mite-plant and predatormite interactions. Bull. OLLB SROP, 30 (5): 9.

Sallam, Gihan M.; Yassin, E. M. A. and Abd El-Azeim, Nahla A., 2007. The predatory insects, mites and spiders of $P$. dactylifera in 
Rashid region, El-Beheira Governorate, Egypt. $1^{\text {st }}$ international conference of $P$. dactylifera, integrated crop management of $P$. dactylifera and its impacts for producing clean and safety dates, September 2007 Giza, Egypt: 10.
Walter, D. E. and O'Dowd, D. J., 1995. Life on the forest phylloplane: hairs, little houses, and myriad mites. In Forest Canopies (M.D. Lowman and N.M. Nadkarni, ed.) Academic Press, San Diego: 325-351. 